\title{
Diabetic dermopathy
}

\author{
SUSANNAH MC GEORGE', SHERNAZ WALTON²
}

\begin{abstract}
Diabetic dermopathy is a term used to describe the small, round, brown atrophic skin lesions that occur on the shins of patients with diabetes. The lesions are asymptomatic and occur in up to $55 \%$ of patients with diabetes, but incidence varies between different reports. Diabetic dermopathy is more common in older patients and those with longstanding diabetes. It is associated with other microvascular complications of diabetes such as retinopathy, nephropathy and neuropathy and also with large vessel disease. Histological changes include epidermal atrophy with flattening of the rete ridges, dermal fibroblastic proliferation, altered collagen, dermal oedema and an increase in dermal capillaries, with a perivascular inflammatory infiltrate, changes to the vessel walls and melanin and haemosiderin deposition. The underlying mechanism for diabetic dermopathy is unknown, although it may be related to local thermal trauma, decreased blood flow causing impaired wound healing or local subcutaneous nerve degeneration. Diabetic dermopathy requires no treatment, but may be a surrogate for more serious complications of diabetes, which require investigation and management.
\end{abstract}

Br J Diabetes Vasc Dis 2014;14:95-97

Key words: dermatology, diabetes mellitus, diabetic dermopathy, microvascular disease, akin

\section{Introduction}

Diabetic dermopathy is the commonest skin condition that occurs in patients with diabetes mellitus. The condition was first reported in 1964 by Melin, who described small, circumscribed, brownish atrophic skin lesions occurring on the lower extremities. ${ }^{1}$ The phrase diabetic dermopathy was coined by Binkley in $1965,{ }^{2}$ but the lesions have variously been termed "pigmented pretibial patches", 3 "shin spots", 4 "spotted leg syndrome" 5 and

Department of Dermatology, Brighton and Sussex University Hospitals NHS Trust, Brighton, UK

2 Department of Dermatology, Hull and East Yorkshire Hospitals NHS Trust and Hull York Medical School, Hull, UK

Address for correspondence: Dr Susannah George Department of Dermatology, Brighton General Hospital, Elm Grove, Brighton, BN2 3EW, UK

Tel: +44 (0)1273 696955 ext 5019

E-mail: susannah@susannahgeorge.net

http://dx.doi.org/10.15277/bjdvd.2014.027 "diabetic dermangiopathy". ${ }^{6}$ In his original clinical description, Melin concluded that they were more or less specific for diabetes mellitus ${ }^{1}$ and, while most reports published since then agree with his findings, other authors suggest that the lesions may be seen in patients without diabetes. ${ }^{4}$ One study found that they occurred in $1.5 \%$ of non-diabetic medical students and in $20.2 \%$ of non-diabetic controls, derived from the endocrine clinic population. ${ }^{4}$ It has been suggested that at least four lesions are characteristic of diabetes. ${ }^{7}$

Diabetic dermopathy has been reported to occur in between $0.2-55 \%$ of patients with diabetes. ${ }^{14,7-11}$ The lowest incidence was reported in a study from India of 500 patients with diabetes $(98.8 \%$ type 2 diabetes), in which only one patient $(0.2 \%)$ was found to have diabetic dermopathy. ${ }^{10}$ The reason suggested for the low incidence reported in most studies from India is that the skin lesions may be more difficult to see in individuals with darker skin types. ${ }^{12}$ However, a notable exception is a study from the Western Himalayas in which diabetic dermopathy was identified in $36 \% .{ }^{9}$

Diabetic dermopathy is more common in patients older than 50 years ${ }^{13}$ and in those with a longer duration of diabetes. ${ }^{1,4,13}$ In older patients diabetic dermopathy was found to occur after a shorter duration of diabetes, whereas in younger patients lesions occurred only after ten years in patients less than 20 years of age. ${ }^{1}$ The lesions have been reported to occur more frequently in male patients, ${ }^{1,4}$ however, this difference is not always significant ${ }^{13}$ and other studies have not found any difference..$^{14}$ It is uncertain as to whether diabetic dermopathy is more common in patients with type 1 or type 2 diabetes. ${ }^{12}$

\section{Diagnosis}

\section{i.Clinical findings}

The lesions are asymptomatic and there are only a few reports of their onset and progression. 1,2 The lesions begin spontaneously as non-blanching, scaly, red or purple, round or oval macules or papules. There may be induration, with a central depression or vesiculation (Figure 1). ${ }^{2}$ These lesions subsequently progress to the characteristic scar-like lesions of diabetic dermopathy. ${ }^{1}$ In established lesions there may be a thin keratin scale. ${ }^{2}$ Most frequently presenting as round, brown, atrophic lesions of less than $1 \mathrm{~cm}$ diameter, some may be elongated and up to $2.5 \mathrm{~cm}$ (Figure 2). 3,15 They usually occur on the shins in a bilateral asymmetrical distribution, but have been rarely reported on the arms, thighs, trunk and abdomen. ${ }^{6,16}$ The intensity of pigmentation corresponds to the degree of atrophy, with the darkest lesions also being the most atrophic. ${ }^{1}$ Each individual 
Figure 1. Early red/purple lesions showing depressed centre and vesiculation

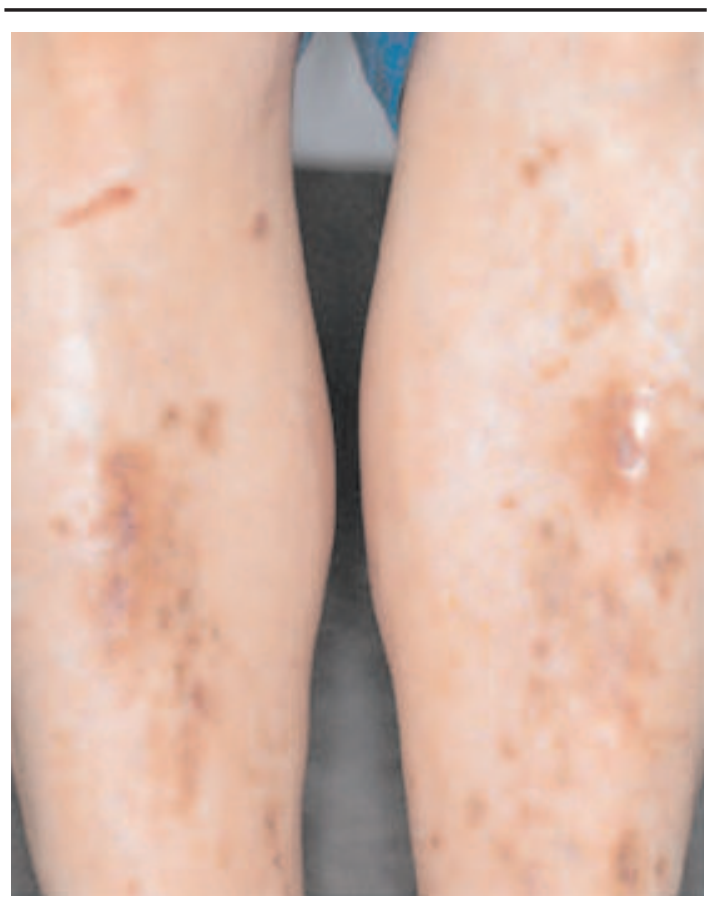

Copyright permission obtained from Dr Niels Veien, D@nderm

Figure 2. Brown macules with atrophic scars on the shins of a diabetic patient

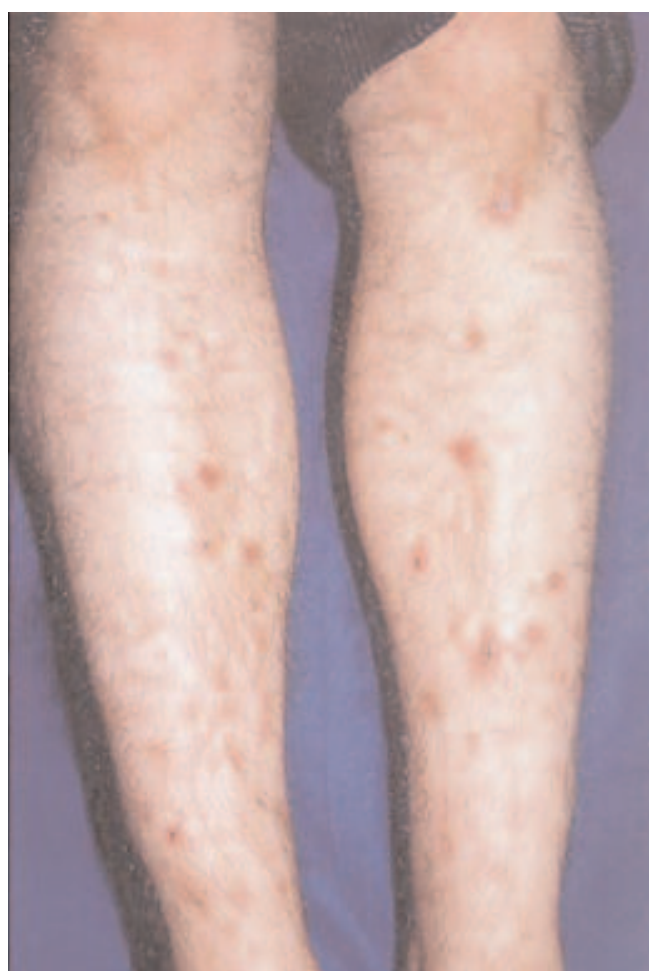

lesion lasts on average 18-24 months, before fading to minimally atrophic macules, or clearing completely. ${ }^{1}$ In some cases, the brownish colour disappears and is replaced by a slight depigmentation. ${ }^{1}$ As older lesions clear, new lesions appear. ${ }^{1}$

\section{ii.Clinical associations}

The presence of diabetic dermopathy may be an indicator of other more serious pathology and its occurrence has been associated with both microvascular complications ${ }^{1,13}$ and large vessel disease. ${ }^{14}$ In Melin's original study $69 \%$ of patients with skin lesions had retinopathy; only $25 \%$ of the group without skin lesions had retinopathy. ${ }^{1}$ Similarly, there were significantly more patients with nephropathy in the group with diabetic dermopathy, than in those without (27\% versus $5 \%$ ) and more patients with neuropathy amongst those with diabetic dermopathy than in those without (57\% versus 20\%). ${ }^{1}$ Another study examining the relationship between retinopathy and diabetic dermopathy also found that the frequency of retinopathy in patients with diabetic dermopathy was significantly greater than in patients without diabetic dermopathy (44\% versus 15\%). ${ }^{17}$ Although most other studies have confirmed similar findings, one study of 457 diabetic patients found that diabetic dermopathy was associated with neuropathy, but not retinopathy or nephropathy. ${ }^{14}$ Shemer et al. (1998) found that patients with other diabetic microvascular complications such as retinopathy, neuropathy and nephropathy were more likely to have diabetic dermopathy than those patients with no complications. ${ }^{13}$ Furthermore, the incidence of diabetic dermopathy increased with number of complications, such that diabetic dermopathy occurred in $21 \%$ of those with no microvascular complications, $52 \%$ of those with one complication, $57 \%$ of those with two complications and $81 \%$ of those with all three complications. ${ }^{13}$

Surprisingly, no correlation between the presence of diabetic dermopathy and $\mathrm{HbA}_{1 \mathrm{c}}$ was found. ${ }^{14}$

\section{iii. Histopathology}

Diagnosis of diabetic dermopathy is usually made by clinical examination. ${ }^{12}$ Although frequently recognised, lesions of diabetic dermopathy are rarely biopsied ${ }^{12,18}$ due to concerns about poor healing on the lower limbs. Biopsies were performed in early studies, with further information derived from post mortem specimens. ${ }^{18}$

Epidermal features include atrophy or flattening with obliteration of the rete ridges, hyperkeratosis and variable pigmentation in the basal cells. ${ }^{1,2,19}$

Dermal changes include fibroblastic proliferation, increased density of the collagen, with thickening of the collagen bundles and fragmentation or separation of the collagen fibres and dermal oedema. ${ }^{1,2}$ There are more fine wavy elastic fibres than in the skin of non-diabetics. ${ }^{19}$ There are increased numbers of capillaries with hyalinization of dermal arterioles, endothelial proliferation, and narrowing or partial occlusion of the vessel lumina and deposition of periodic-acid-Schiff positive material in their walls. ${ }^{6}$ There is increased dermal melanin and haemosiderin deposition. ${ }^{18}$

\section{Causes}

The underlying mechanism behind diabetic dermopathy is unknown, although many theories have been suggested. As the lesions are asymptomatic, patients often do not notice their appearance and may assume they have arisen due to trauma. ${ }^{2}$ Melin explored the theory that the lesions of 


\section{Key messages}

- Diabetic dermopathy manifests as small, round, brown atrophic skin lesions on the shins of patients with diabetes

- The underlying mechanism for diabetic dermopathy is unknown

- The skin lesions are asymptomatic and require no treatment, but are associated with other microvascular complications and large vessel disease

diabetic dermopathy occurred secondary to trauma; however attempts to experimentally reproduce the lesions by striking the skin with a rubber hammer were unsuccessful. ${ }^{1}$ Another theory is that the lesions were caused by areas of relative ischaemia caused by changes in local temperature. Binkley suggested that the predilection for the shins was due to decreased skin temperature, slow blood flow, increased plasma viscosity and vessel fragility. ${ }^{2}$ Experimental attempts to induce diabetic dermopathy in patients with diabetes revealed that application of thermal stimuli, both hot and cold, induced atrophic circumscribed skin lesions in elderly patients with diabetes, or those with diabetes of long duration. However, similar lesions were also elicited in the skin of patients with amyloidosis, suggesting that they were not specific for diabetes. ${ }^{16}$ Local ischaemia was postulated as a possible cause for diabetic dermopathy, but blood flow in the lesions has been shown to be increased, rather than decreased, making this theory less likely. ${ }^{20}$ The authors concluded that diabetic dermopathy does not occur due to ischaemia and represents a scarring process due to defective wound healing. ${ }^{20} \mathrm{~A}$ subsequent study confirmed increased blood flow in the actual lesions, but found decreased blood flow in normal appearing skin on the shins of patients with diabetic dermopathy. The authors proposed that the decreased blood flow predisposed patients with diabetes to inadequate wound healing, which led to the formation of the characteristic lesions of diabetic dermopathy. ${ }^{21}$ Another suggested mechanism is that diabetic neuropathy causes subcutaneous nerve degeneration, which leads to diabetic dermopathy. ${ }^{22}$

\section{Management}

The lesions of diabetic dermopathy are asymptomatic and no treatment is recommended or has been shown to be effective. Cosmetic camouflage may be used to disguise the appearance of the skin lesions if required. However, given the strong association of diabetic dermopathy with other microangiopathic complications of diabetes, the lesions might be considered a surrogate for more serious pathology. The finding of skin lesions with the appearance of diabetic dermopathy in patients not previously known to have diabetes should prompt investigations for the condition. ${ }^{4}$

Given the association with other microvascular complications, optimisation of glycaemic control in patients with diabetic dermopathy to minimise the progression of retinopathy, nephropathy and neuropathy is paramount.

\section{Conflict of interest None \\ Funding sources None}

\section{References}

1. Melin H. An atrophic circumscribed skin lesion in the lower extremities of diabetics. Acta Med Scand 1964;176(Suppl.423):9-75.

2. Binkley GW. Dermopathy in the diabetic syndrome. Arch Dermatol 1965;92:625-34.

http://dx.doi.org/10.1001/archderm.1965.01600180017003

3. Bauer MF, Levan NE, Frankel A, Bach J. Pigmented pretibial patches. A cutaneous manifestation of diabetes mellitus. Arch Dermatol 1966;93:2826. http://dx.doi.org/10.1001/archderm.1966.01600210018003

4. Danowski TS, Sabeh G, Sarver ME et al. Shin spots and diabetes mellitus Am J Med Sci 1966;251:570-5.

http://dx.doi.org/10.1097/00000441-196605000-00011

5. Murphy RA. Skin lesions in diabetic patients: The "spotted-leg" syndrome. Lahey Clin Found Bull 1965;14:10-4.

6. Bauer M, Levan NE. Diabetic dermangiopathy. A spectrum including pigmented pretibial patches and necrobiosis lipoidica diabeticorum. $\mathrm{Br} J$ Dermatol 1970:83:528-35. http://dx.doi.org/10.1111/j.1365-2133.1970.tb15736.x

7. Ngo BT, Hayes KD, DiMiao DJ, et al. Manifestations of cutaneous diabetic microangiopathy. Am J Clin Dermatol 2005;6:225-37. http://dx.doi.org/10.2165/00128071-200506040-00003

8. Mahajan S, Koranne RV, Sharma SK. Cutaneous manifestation of diabetes mellitus. Indian J Dermato/ Venereo/ Leprol 2003;69:105-08.

9. Goyal A, Raina S, Kaushal SS et al. Pattern of cutaneous manifestations in diabetes mellitus. Indian J Dermato/ 2010;55:39-41. http://dx.doi.org/10.4103/0019-5154.60349

10. Ragunatha $S$, Anitha B, Inamadar AC, et al. Cutaneous disorders in 500 diabetic patients attending diabetic clinic. Indian J Dermatol 2011; 56:160-4.

11. Timshina DK, Thappa DM, Agrawal A. A clinical study of dermatoses in diabetes to establish its markers. Indian J Dermatol 2012;57:20-5. http://dx.doi.org/10.4103/0019-5154.92671

12. Morgan AJ, Schwartz RA. Diabetic dermopathy: A subtle sign with grave implications. J Am Acad Dermatol 2008;58:447-51. http://dx.doi.org/10.1016/j.jaad.2007.11.013

13. Shemer A, Bergman R, Linn S, et al. Diabetic dermopathy and internal complications in diabetes mellitus. Int J Dermatol 1998;37:113-15. http://dx.doi.org/10.1046/j.1365-4362.1998.00273.x

14. Romano G, Moretti G, Di Benedetto A, et al. Skin lesions in diabetes mellitus: prevalence and clinical correlations. Diabetes Res Clin Pract 1998;39:101-06. http://dx.doi.org/10.1016/S0168-8227(97)00119-8

15. Port M. Diabetic dermopathy: a controversy in dermatology. J Am Podiatry Assoc 1982;72:418-23. http://dx.doi.org/10.7547/87507315-72-8-418

16. Lithner F. Cutaneous reactions of the extremities of diabetics to local thermal trauma. Acta Med Scand 1975;198:319-25.

17. Abdollahi A, Daneshpazhooh M, Amirchaghmaghi E, et al. Dermopathy and retinopathy in diabetes: is there an association? Dermatology 2007:214:133-6.

18. McCash S, Emanuel PO. Defining diabetic dermopathy. J Dermatol 2011;38:988-92. http://dx.doi.org/10.1111/j.1346-8138.2011.01251.x

19. Fisher ER, Danowski TS. Histologic, histochemical, and electron microscopic features of the shin spots of diabetes mellitus. Am J Clin Pathol 1968; 50:547-54

20. Wigington $G$, Ngo B, Rendell M. Skin blood flow in diabetic dermopathy Arch Dermatol 2004;140:1248-50. http://dx.doi.org/10.1001/archderm.140.10.1248

21. Brugler A, Thompson S, Turner S, Ngo B, Rendell M. Skin blood flow abnormalities in diabetic dermopathy. J Am Acad Dermatol 2011;65:55963. http://dx.doi.org/10.1016/j.jaad.2010.06.010

22. Kiziltan ME, Benbir G, Akalin MA. Is diabetic dermopathy a sign for severe neuropathy in patients with diabetes mellitus? Nerve conduction studies and symptom analysis. Clin Neurophysiol 2006;117:1862-9 http://dx.doi.org/10.1016/j.clinph.2006.05.007 